\title{
Massive young stellar objects in the N66/NGC 346 region of the SMC ${ }^{\star}$
}

\author{
M. Rubio ${ }^{1}$, R. H. Barbá ${ }^{2}$, and V. M. Kalari ${ }^{1}$ \\ ${ }^{1}$ Departamento de Astronomía, Universidad de Chile, Casilla 36-D Santiago, Chile \\ e-mail: mrubio@das.uchile.cl \\ 2 Departamento de Física, Universidad de La Serena, Benavente 980, La Serena, Chile
}

Received 23 January 2017 / Accepted 21 March 2018

\begin{abstract}
We present $H K$ spectra of three sources located in the N 66 region of the Small Magellanic Cloud. The sources display prominent stellar $\mathrm{Br} \gamma$ and extended $\mathrm{H}_{2}$ emission, and exhibit infrared excesses at $\lambda>2 \mu \mathrm{m}$. Based on their spectral features, and photometric spectral energy distributions, we suggest that these sources are massive young stellar objects. The findings are interpreted as evidence of on-going high mass star formation in N 66.
\end{abstract}

Key words. magellanic clouds - infrared: stars - HII regions - stars: protostars - techniques: spectroscopic

\section{Introduction}

The metal-poor Magellanic Clouds with their unobscured line of sight, their small internal extinction and their profusion of starforming regions, constitute one of the best laboratories to study the interaction between forming massive stars and their environment. In particular, the Small Magellanic Cloud (SMC), having a metallicity $(Z) 1 / 5$ solar metallicity (Russell \& Dopita 1992), allows one to study the process of star formation in a gas-rich environment approaching that of the early Universe. Its proximity, at a distance of $61 \pm 1 \mathrm{kpc}$ (Hilditch et al. 2005), makes it possible to resolve individual stars in a low-metallicity environment, and study star formation in metal-poor environments (Kalari \& Vink 2015; Ward et al. 2017; Kalari et al. 2018).

N 66 is the brightest H II region in the SMC (Henize 1956) and is located in the northeast of the SMC bar. It is excited by the dense cluster of massive young stars, NGC 346. Massey et al. (1989) performed an extensive study of the stellar content of this cluster, also referred to as N66A, and identified at least 33 O-type stars, including 11 of spectral type O6.5 or earlier. Twenty-two of these $\mathrm{O}$ stars are contained in NGC 346 and the others are isolated or in small groups. Multi-wavelength studies of $\mathrm{N} 66$ have shown the complexity and richness of this region. Contursi et al. (2000) showed mid-infrared (mid-IR) emission peaks coincident with the main features of the ionized gas. Using high-sensitivity CO observations, Rubio et al. (2000) detected weak molecular gas towards the $\mathrm{H}$ II region associated to compact dense molecular hydrogen knots from emission in the $v(1-0) \mathrm{S}(1)$ line of $\mathrm{H}_{2}$ at $2.12 \mu \mathrm{m}$. These data revealed a beautiful photodissociation region with molecular gas not yet photo-dissociated by the UV radiation of the stars, concentrated in dense clumps with a very small surface filling factor. In addition, Rubio et al. (2000) discovered several embedded stars

\footnotetext{
* Based on observations collected at the European Organisation for Astronomical Research in the Southern Hemisphere under ESO programme 063.C-0329(A) and 072.C-0466(A).
}

and/or star clusters, and suggested that three successive stellar generations have already formed in less than 3 million years.

Studies of the stellar population done with HST/ACS observations by Nota et al. (2006), Gouliermis et al. (2006), Sabbi et al. (2007), and Schmeja et al. (2009) have found a large number of pre-main sequence (PMS) stars with ages of 3-5 Myrs in N 66. Dedicated Spitzer studies have uncovered massive young stellar objects (mYSOs) in the region (Simon et al. 2007). The spatial distribution of optical and infrared (IR) stellar populations shows a concentration of stars coincident with the neutral molecular clumps identified by Rubio et al. (2000) from $\mathrm{CO}(2-1)$ observations. The multi-wavelength photometric studies strengthened the suggestion that the region may have sites of recent or ongoing star formation, and that the newly formed stars are still embedded in the molecular cloud and thus invisible at optical wavelengths.

In this paper, we present $H$ and $K$ band spectroscopy of three early-type stars in N66 in the SMC. These three stars represent the best candidate mYSOs in N66 based on their near-infrared (NIR) $J H K$ s colours. They are bright and have the largest IR excess of the sample. The NIR spectral observations complement the multi-wavelength photometric studies of YSOs conducted in this region, and help gain a more detailed understanding of their nature, and differences with respect to Galactic counterparts.

This paper is organised as follows. Section 2 contains a description of the photometric and spectroscopic observations of our mYSOs. Section 3 presents our results from these observations. A discussion pertaining to the nature and formation scenario of the mYSOs is given in Sect. 4. Finally, we present our conclusions in Sect. 5.

\section{Observations}

\subsection{Near-infrared photometry and target selection}

We obtained deep NIR $J H K_{\mathrm{s}}$ images of N 66 using the Infrared Spectrometer And Array Camera (ISAAC) imager mounted on 


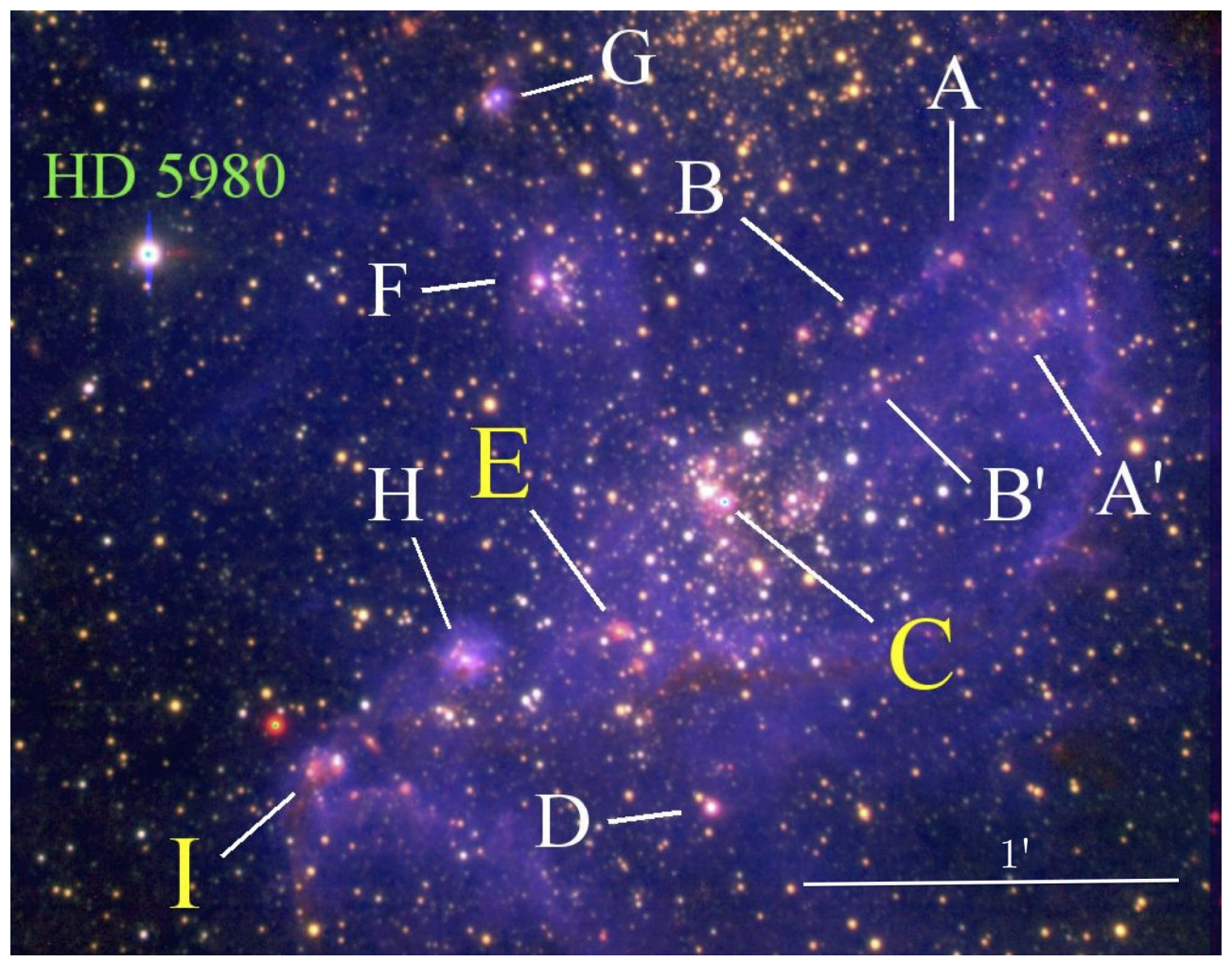

Fig. 1. Colour composite image of N66. In red and green channels are $K_{s^{-}}$and $J$-band images obtained with VLT/ISAAC, respectively. The blue channel is a VLT/FORS1 archival $\mathrm{H} \alpha$ image. Indicated in the images are the ISO sources labelled A to $\mathrm{H}$ (Rubio et al. 2000; Contursi et al. 2000). Yellow labels $\mathrm{C}, \mathrm{E}$, and I indicate the NIR sources of this study. North is up, and east is to the left. The scale-bar in the lower-left corner gives 1 arcmin.

the Antu $8.2 \mathrm{~m}$ Very Large Telescope (VLT) at Paranal Observatory. We used the short-wavelength arm equipped with a $1024 \times 1024$ pixels Hawaii Rockwell array, with a pixel scale of 0 .'148 pix $^{-1}$ and a total field of view (FOV) of about $2.5 \times 2.5$. The observations were done in a series of six frames, each individual frame with $10 \mathrm{~s}$ integration time in each filter. The median seeing of our observations was 0 ". 8 . The individual frames were coadded and a $60 \mathrm{~s}$ image was stored. We observed two fields with an overlap of about $30^{\prime \prime}$ to cover most of the N 66 region. In each filter, we interleaved sky frame observations for every 10 mins of on-source imaging. These sky frames were chosen in a field with faint stars and no extended emission at $300^{\prime \prime}$ South of the position of N66. The sky field was observed in a similar way to the source frames. The procedure was repeated several times to achieve a final integration time of $3600 \mathrm{~s}$ in $J, H$, and $K_{s}$.

Previous to the VLT observations, we conducted NIR $J H K_{s}$ imaging of $\mathrm{N} 66$ using the 2.5-m Du Pont Telescope at Las Campanas Observatory with the $256 \times 256$ NICMOS III Camera, ClassicCAM (Persson et al. 1992). These images covered a $1^{\prime} \times 1$.2 area with a pixel scale of 0. . $35 \mathrm{pix}^{-1}$ and typical seeing of 0 ". 8 in $K_{s}$. The observation consisted of a series of ten frames, each individual frame with an integration time of $20 \mathrm{~s}$ per filter. Sky frames in each filter were taken in a field at 300" South with faint stars and no extended emission. The sky field was observed in a similar way to the source frames. This observing procedure was repeated to produce a nine-position mosaic with separation of $20^{\prime \prime}$. The total integration time in each filter resulted in $1800 \mathrm{~s}$ in $K_{s}, 1800 \mathrm{~s}$ in $H$, and $2000 \mathrm{~s}$ in $J$.

To produce the final images, for both LCO and VLT observations, each image was dark corrected, flat-fielded, and sky subtracted, and then median combined using IRAF ${ }^{1}$ procedures. The final images were aligned with respect to the $K_{s}$ image by

1 IRAF is distributed by the National Optical Astronomy Observatory, which is operated by the Association of Universities for Research in means of several common stars. The final VLT image, which combines the two overlapping fields cover a total $2.5 \times 5.0$ area. The resulting LCO image, of only $65^{\prime \prime} \times 76^{\prime \prime}$ size includes the NGC 346 cluster and the ISO peaks E and I (Contursi et al. 2000). Figure 1 shows a composite $\mathrm{H} \alpha, J, K_{s}$ image of the central 3.4 $\times 2 ! 7$ area of N 66 produced with the $K_{s}$ and $J$ images obtained at VLT and a FORS/VLT H $\alpha$ image retrieved from the ESO archive, as red, green, and blue channels, respectively. Several IR sources appear very bright in this composite image. These sources are located towards the $\mathrm{H}_{2}$ knots and $7 \mu \mathrm{m}$ peaks associated with $\mathrm{CO}(2-1)$ emission from molecular clouds (Rubio et al. 2000; Contursi et al. 2000).

We performed point spread function (PSF) photometry on ISAAC/VLT images using DAOPHOT (Stetson 1987) incorporated in IRAF, reaching a limiting magnitude of about $K_{s}=22$, $H=21$ and $J=21$, respectively. The photometric calibration was made with respect to $2 \mathrm{MASS}$ photometry. We choose the 2MASS system since it resembles the ISAAC and LCO NIR filters. We identified 20 bright stars outside the central cluster which were common to the LCO, ISAAC, and 2MASS images. We then applied offsets determined with respect to the 2MASS photometry to the LCO and ISAAC photometry. The brightest IR sources appear saturated in the VLT images, therefore we performed our own photometry and used the LCO $J H K_{s}$ images to measure the IR magnitudes of these brightest sources instead of using Gouliermis et al. (2010) photometry. Table 1 gives the $J H K_{s}$ magnitudes obtained for the three selected objects. In the top panel of Fig. 2, we show a colour-magnitude diagram of the stars of the region shown in Fig. 1 and brighter than $J=20$, obtained from photometry performed on the VLT images. The lower panel of the same figure shows the $(J-H)$ vs. $(H-K \mathrm{~s})$ colour-colour diagram.

Astronomy (AURA) under a cooperative agreement with the National Science Foundation. 
Table 1. NIR magnitudes of mYSOs in N 66.

\begin{tabular}{cccccccccc}
\hline \hline Source & $\operatorname{RA}(2000)$ & $\operatorname{DEC}(2000)$ & $\mathrm{J}$ & $e_{J}$ & $H$ & $e_{H}$ & $K s$ & $e_{K s}$ & Rubio et al. (2000) \\
\hline 1 & 005905.43 & -721035.5 & 14.44 & 0.02 & 13.50 & 0.04 & $12.05(\mathrm{~L})$ & 0.05 & $\mathrm{C}$ \\
2 & 005909.29 & -721057.4 & 16.37 & 0.03 & 14.66 & 0.02 & $12.86(\mathrm{~L})$ & 0.01 & $\mathrm{E}$ \\
3 & 005919.66 & -721119.6 & 16.01 & 0.03 & 15.61 & 0.04 & 15.15 & 0.04 & $\mathrm{I}$ \\
\hline
\end{tabular}

Notes. The letter $\mathrm{L}$ in parentheses denotes those magnitudes obtained from photometry performed on the LCO/du Pont images; these sources appear saturated in the ISAAC/VLT $K_{s}$ images.

\subsection{Infrared spectroscopy}

We selected the three brightest sources for $K$-band spectroscopy based on the NIR excess from the images obtained with ClassicCAM attached to the Du Pont telescope at Las Campanas Observatory and with ISAAC/VLT at Paranal Observatory. Figure 2 shows the sources which correspond spatially to the emission peaks designated C, E, and I in Contursi et al. (2000).

We obtained low-dispersion $(R \sim 1000) H K$-band spectra of these latter three sources in N66 in the SMC using the SofI instrument at the NTT at La Silla Observatory. The total integration time on each source was $1200 \mathrm{~s}$ and the signal-to-noise ratio $(\mathrm{S} / \mathrm{N})$ obtained ranged from 30 to 80 . Sky selection for background subtraction was crucial as all the selected sources were embedded in bright nebulosity and crowded fields. The observations were conducted in an "ABBA" sequence. Darks and flat-field calibrations were obtained for each night along with several spectrophotometric standards to get flux calibrations. Telluric correction was performed using the standards Hip4536 or Hip7806 (F5V and F7V spectral types respectively), taken immediately before and after the target, at similar airmass. The velocity is given in heliocentric rest frame. The NIR spectra of the sources are shown in Fig. 3. The spectra of sources $\mathrm{C}$ and $\mathrm{E}$ were obtained along the slit at the same time with a slit positionangle of $P A=39^{\circ}$. The spectra of source I were obtained with the slit in $P A=59^{\circ}$.

\subsection{Archival photometry}

In addition to the IR photometry campaign, we used the archival Hubble imaging study of Sabbi et al. (2007) to find optical counterparts in the filters F555W (hereafter $V$ ) and F814W (hereafter $I$ ). For the stars $\mathrm{C}$ and $\mathrm{I}$, we find cross matches within a radius of 0 '. 1 , acceptable for the crowding and confusion in this region. In the 0 ".06 HST resolution images, there are two stars associated to our Source C. These stars are visible in $V$ and their HST VI photometry show two roughly similar brightness stars in $V I$ within 0 ". 5 of the source C, catalogued by Sabbi et al. (2007) with identification numbers (IDs) in that paper of ID152 and ID168 with V magnitude of 16.62 and 16.71, respectively. We select ID152 as our Source C counterpart, since it falls within the cross match radius $(0.5 \times$ the seeing of the infrared imaging) of the Source $\mathrm{C}$ infrared coordinates. The IR photometry of Source $\mathrm{C}$ includes a contribution from both HST sources. For Source E, the nearest optical neighbour is $>0{ }^{\prime \prime} .15$ and does not seem to be a physical counterpart of the NIR source.

We used the HST H $\alpha$ image to check for emission line stars and compact HII regions and we cross match the stars with midIR Spitzer (Simon et al. 2007) and Herschel public catalogues (Seale et al. 2014). We have checked that their are no neighbours falling within the FWHM of the imaging at mid-IR wavelengths, thereby contaminating the determined magnitudes. The photometry of the Spitzer IRAC $3.6-8 \mu \mathrm{m}$ bands are given in Table 2,
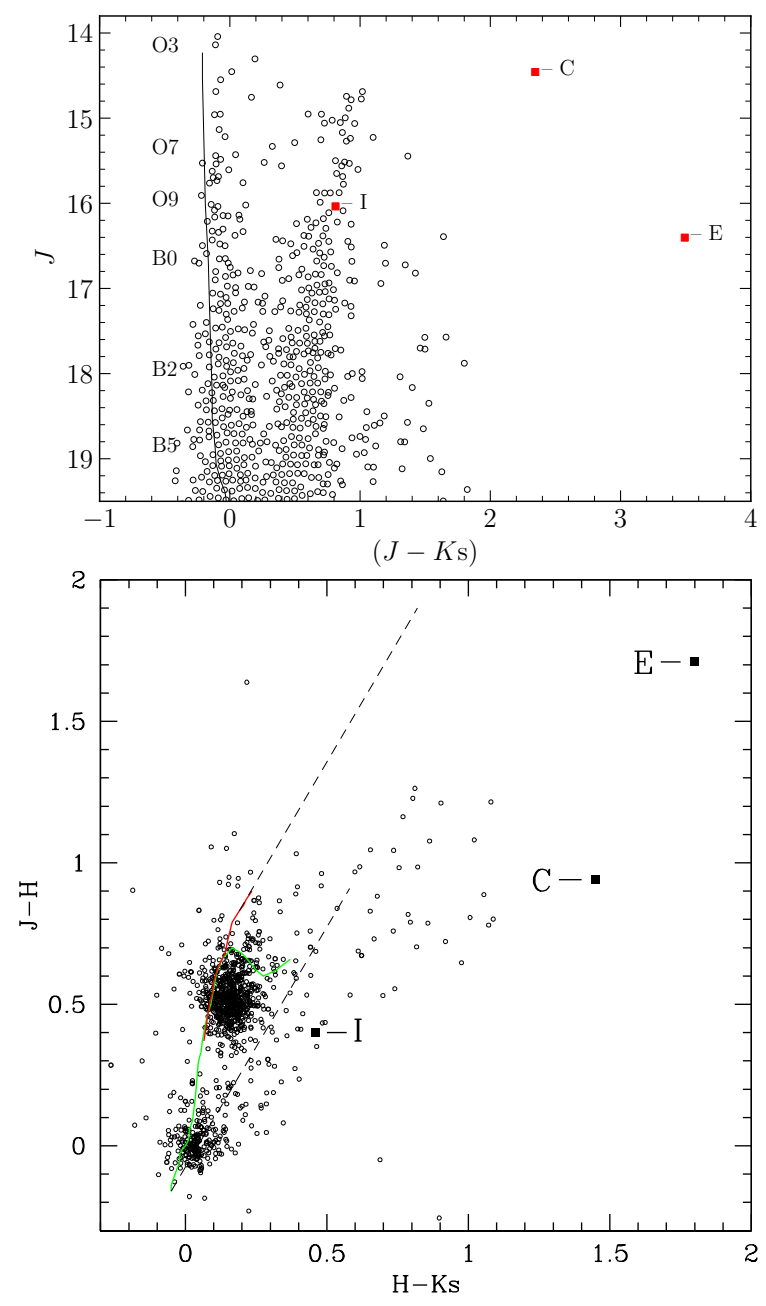

Fig. 2. VLT/ISAAC NIR colour-magnitude and colour-colour diagrams of N 66, with the positions of the selected sources for spectroscopy marked. For sources $\mathrm{C}$ and $\mathrm{E}$, the $K \mathrm{~s}$ magnitudes are from LCO observations. The three sources correspond to the brightest infrared excess sources in their respective molecular clouds. The green and red solid lines in the colour-colour diagram are the dwarf and giant locus respectively. The reddening track of a normal O3 V star and Cool Giant G is plotted as dashed black lines. The ZAMS between B5 and O3 at the distance of the SMC is indicated with a solid black line.

while the fluxes of the long-wavelength Spitzer MIPS 24-70 $\mu \mathrm{m}$, and Herschel $100-350 \mu \mathrm{m}$ photometry is given in Table 3 .

\section{Results}

\subsection{Photometry}

The three IR sources for which we have obtained spectra are plotted and labelled in the $\left(J-K_{s}, K_{s}\right)$ colour-magnitude diagram 

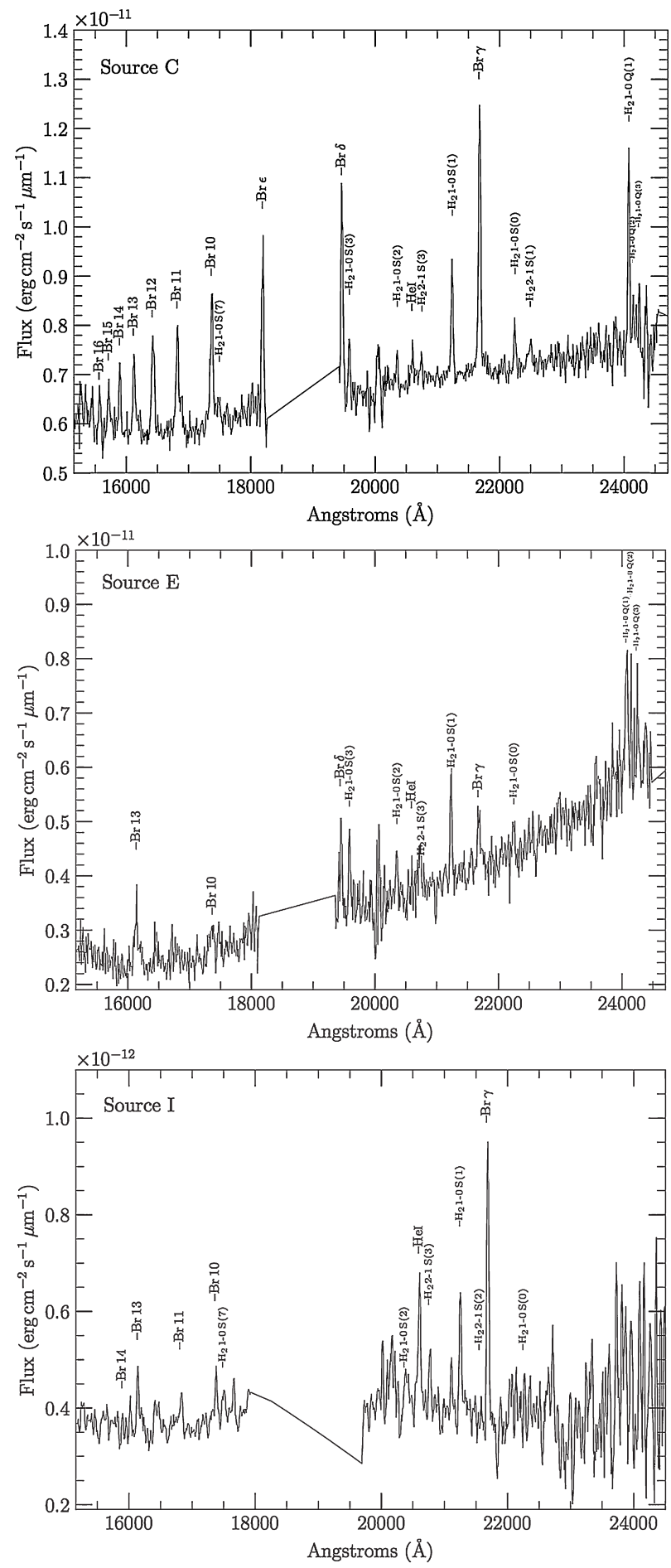

Fig. 3. $H K$ spectra of each star as labelled. Prominent $\mathrm{H}$ Brackett series, $\mathrm{He}$, and $\mathrm{H}_{2}$ emission lines are seen.

in Fig. 2. In this diagram, the solid line represents the location of the zero-age main sequence (ZAMS; Hanson et al. 1997), using a distance modulus of 18.9 to the SMC and assuming no reddening (Keller \& Wood 2006). Source C is one of the most luminous IR sources in NGC 346 (after WR/LBV system HD 5980) with $K_{s}=12.05$, and source E displays the reddest colour with a $J-K_{s}=3.4$. Both sources show IR colours distinct to those of the majority of the stars in the field and consistent with their different nature. Sources $\mathrm{C}$ and $\mathrm{E}$ lie in the region of the colour-colour diagram of IR excess (see Fig. 2) and are similar to the brightest IR sources detected in 30 Doradus in the
Table 2. Archival optical and NIR photometry.

\begin{tabular}{cccc}
\hline \hline Filter & $\begin{array}{c}\text { Source C } \\
(\mathrm{mag})\end{array}$ & $\begin{array}{c}\text { Source E } \\
(\mathrm{mag})\end{array}$ & $\begin{array}{c}\text { Source I } \\
(\mathrm{mag})\end{array}$ \\
\hline $\mathrm{F} 555 \mathrm{~W}^{1}$ & $16.617 \pm 0.009$ & - & $16.919 \pm 0.011$ \\
$\mathrm{~F} 814 \mathrm{~W}^{1}$ & $15.983 \pm 0.008$ & - & $16.865 \pm 0.012$ \\
& $\left(0^{\prime \prime} .065\right)^{*}$ & - & $\left(0^{\prime \prime} 054\right)^{*}$ \\
$3.6 \mu \mathrm{m}^{2}$ & $10.101 \pm 0.018$ & $10.063 \pm 0.014$ & $12.842 \pm 0.179$ \\
$4.5 \mu \mathrm{m}^{2}$ & $9.422 \pm 0.013$ & $8.861 \pm 0.016$ & $12.142 \pm 0.034$ \\
$5.8 \mu \mathrm{m}^{2}$ & $8.714 \pm 0.015$ & $7.824 \pm 0.009$ & $10.391 \pm 0.035$ \\
$8.0 \mu \mathrm{m}^{2}$ & $\begin{array}{c}7.234 \pm 0.012 \\
\end{array}$ & $\begin{array}{c}6.811 \pm 0.008 \\
\left(0^{\prime \prime} .039\right)^{*}\end{array}$ & $\begin{array}{c}8.867 \pm 0.047 \\
\left(0^{\prime \prime} 067\right)^{*}\end{array}$ \\
& & & $\left(0^{\prime \prime} .416\right)^{*}$ \\
\hline
\end{tabular}

Notes. ${ }^{(1)}$ Sabbi et al. 2007; (2) Seale et al. 2014; ${ }^{(*)}$ Denotes the separation in arcsec of our source positions.

Table 3. Archival mid-IR photometric fluxes.

\begin{tabular}{cccc}
\hline \hline Filter $^{1}$ & $\begin{array}{c}\text { Source C } \\
(\mathrm{mJy})\end{array}$ & $\begin{array}{c}\text { Source E } \\
(\mathrm{mJy})\end{array}$ & $\begin{array}{c}\text { Source I } \\
(\mathrm{mJy})\end{array}$ \\
\hline $24 \mu \mathrm{m}$ & $2154 \pm 8.48$ & $944.5 \pm 4.13$ & $425.5 \pm 4.3$ \\
$70 \mu \mathrm{m}$ & $3766 \pm 51.51$ & - & $5598 \pm 55.33$ \\
$100 \mu \mathrm{m}$ & $1695 \pm 225.9$ & $2400 \pm 208.7$ & $3083 \pm 214.5$ \\
$160 \mu \mathrm{m}$ & $1724 \pm 199.3$ & $1461 \pm 181.9$ & $2702 \pm 213.6$ \\
$250 \mu \mathrm{m}$ & $822.1 \pm 57.74$ & $502.8 \pm 50.12$ & $1118 \pm 46.24$ \\
$350 \mu \mathrm{m}$ & $302.1 \pm 48.99$ & $442.9 \pm 53.73$ & $448 \pm 39.20$ \\
& $\left(22^{\prime \prime} 14\right)^{*}$ & $(0 . .487)^{*}$ & $\left(11^{\prime \prime} 083\right)^{*}$ \\
\hline
\end{tabular}

Notes. ${ }^{(1)}$ Seale et al. 2014; ${ }^{(*)}$ Denotes the separation in arcsec of our source positions.

LMC (Rubio et al. 1998). Source C has an absolute Ks magnitude of $M_{K s}=-6.88$, Source E, $M_{K s}=-6.07$, and Source I $M_{K s}=-3.78$, assuming no reddening to determine their absolute magnitudes. If they do suffer any extinction then they will become even brighter.

According to the distribution of massive YSOs in the $\left(J-K_{s}, K_{s}\right)$ colour-magnitude diagram (Fig. 1 in Bik et al. 2006) sources $\mathrm{C}$ and $\mathrm{E}$ are extreme bright objects comparable in brightness to those of the Galactic massive YSOs in W31 (Blum et al. 2001) or NGC 2024 (Lenorzer et al. 2004), and S106-IRS4 (Felli et al. 1984), and much brighter than Herbig Be stars (Eiroa et al. 2002; Kalari et al. 2014). Therefore, they are extreme bright YSOs. Both sources have magnitudes $K_{s}$ similar to the mYSOs observed in the LMC by Oliveira et al. (2006): N 157B S1, and N $105 \mathrm{~S} 1$, with $K_{s} 11.58$ and 13.31 magnitude, respectively, or for IRAS $05328-6827$ with $K_{s}=11.98$ (van Loon et al. 2005). For $\mathrm{N} 66$ in the SMC, there is very little NIR spectroscopic information for mYSOs. $L$ band spectroscopy for three mYSO candidates has been reported by van Loon et al. (2008) one of which, IRAS $01039-7305$ has a $K_{s}=11.69$ magnitude similar to Source C.

\subsection{NIR spectral features}

We have identified the emission lines in the NIR spectra of each source. These are given in Table 4 for Source C, Table 5 for Source E and Table 6 for source I. In the tables we give the wavelength, flux and equivalent width (EW) for each identified line. We describe the spectral features of the three sources below. Source C. The spectra of Source C (Fig. 3) suggest an embedded star within a compact $\left(\lesssim 5^{\prime \prime}\right)$ nebulosity. In the two-dimensional long-slit image, we find extended emission centred on the source, 
M. Rubio et al.: Massive young stellar objects in the N 66/NGC 346 region of the SMC

Table 4. Identified lines of source C.

\begin{tabular}{|c|c|c|c|}
\hline Line & $\begin{array}{c}\lambda \\
(\mu \mathrm{m})\end{array}$ & $\begin{array}{c}\text { Flux } \\
\left(\mathrm{erg} \mathrm{cm}^{-2} \mathrm{~s}^{-1}\right)\end{array}$ & $\begin{array}{l}\text { EW } \\
(\AA)\end{array}$ \\
\hline Br19 & 15264.7 & $2.3 \times 10^{-15}$ & -3.8 \\
\hline Br18 & 15346.0 & $1.5 \times 10^{-15}$ & -2.4 \\
\hline Br17 & 15443.1 & $2.1 \times 10^{-15}$ & -3.5 \\
\hline Br16 & 15560.7 & $3.4 \times 10^{-15}$ & -3.4 \\
\hline Br15 & 15705.0 & $3.4 \times 10^{-15}$ & -5.8 \\
\hline Br14 & 15884.9 & $4.9 \times 10^{-15}$ & -8.3 \\
\hline Br13 & 16113.7 & $6.7 \times 10^{-15}$ & -11.5 \\
\hline Br12 & 16411.7 & $7.9 \times 10^{-15}$ & -13.6 \\
\hline FeII & 16434.0 & $2.6 \times 10^{-15}$ & -4.5 \\
\hline Br11 & 16811.1 & $1.0 \times 10^{-14}$ & -17.0 \\
\hline FeII & 16877.8 & $3.0 \times 10^{-15}$ & -5.1 \\
\hline Br10 & 17366.7 & $1.34 \times 10^{-14}$ & -22.5 \\
\hline $\mathrm{H}_{2}(1-0) \mathrm{S}(7)$ & 17480.0 & $3.4 \times 10^{-15}$ & -5.6 \\
\hline $\mathrm{Br} 9$ & 18179.1 & $1.27 \times 10^{-14}$ & -20.0 \\
\hline $\mathrm{Pa} 4$ & 18756.1 & $8.02 \times 10^{-14}$ & -134.0 \\
\hline Br8 & 19450.9 & $1.79 \times 10^{-14}$ & -27.7 \\
\hline $\mathrm{H}_{2}(1-0) \mathrm{S}(3)$ & 19576.0 & $4.2 \times 10^{-15}$ & -6.4 \\
\hline $\mathrm{CaI}$ & 19782.0 & $7.0 \times 10^{-16}$ & -1.1 \\
\hline FeII blend & 20041.1 & $5.1 \times 10^{-15}$ & -7.7 \\
\hline $\mathrm{H}_{2}(1-0) \mathrm{S}(2)$ & 20338.0 & $1.8 \times 10^{-15}$ & -2.7 \\
\hline $\mathrm{HeI}$ & 20587.0 & $1.3 \times 10^{-15}$ & -1.9 \\
\hline $\mathrm{H}_{2}(2-1) \mathrm{S}(3)$ & 20730.0 & $1.2 \times 10^{-15}$ & -1.7 \\
\hline $\mathrm{H}_{2}(1-0) \mathrm{S}(1)$ & 21218.3 & $8.4 \times 10^{-15}$ & -11.6 \\
\hline $\mathrm{Br} 7(\mathrm{Br} \gamma)$ & 21661.3 & $2.29 \times 10^{-13}$ & -32.3 \\
\hline $\mathrm{NaI}$ & 22062.4 & $9.0 \times 10^{-16}$ & -1.2 \\
\hline $\mathrm{H}_{2}(1-0) \mathrm{S}(0)$ & 22235.0 & $3.3 \times 10^{-15}$ & -4.7 \\
\hline $\mathrm{H}_{2}(2-1) \mathrm{S}(1)$ & 22477.0 & $5.1 \times 10^{-15}$ & -7.0 \\
\hline $\mathrm{H}_{2}(1-0) \mathrm{Q}(1)$ & 24066.0 & $1.13 \times 10^{-14}$ & -14.6 \\
\hline $\mathrm{H}_{2}(1-0) \mathrm{Q}(2)$ & 24134.0 & $1.8 \times 10^{-15}$ & -2.4 \\
\hline $\mathrm{H}_{2}(1-0) \mathrm{Q}(3)$ & 24237.0 & $2.5 \times 10^{-15}$ & -3.2 \\
\hline
\end{tabular}

Table 5. Identified lines of source E.

\begin{tabular}{lccc}
\hline \hline Line & $\begin{array}{c}\lambda \\
(\mu \mathrm{m})\end{array}$ & $\begin{array}{c}\text { Flux } \\
\left(\mathrm{erg} \mathrm{cm}^{-2} \mathrm{~s}^{-1}\right)\end{array}$ & $\begin{array}{c}\text { EW } \\
(\AA)\end{array}$ \\
\hline $\mathrm{Br} 13$ & 16113.7 & $3.7 \times 10^{-15}$ & -14.8 \\
FeII & 16434.0 & $2.8 \times 10^{-15}$ & -11.6 \\
$\mathrm{Br} 10$ & 17366.7 & $5.6 \times 10^{-15}$ & -21.8 \\
$\mathrm{Br} 8$ & 19450.9 & $5.8 \times 10^{-15}$ & -17.7 \\
$\mathrm{H}_{2}(1-0) \mathrm{S}(3)$ & 19576.0 & $3.9 \times 10^{-15}$ & -11.1 \\
$\mathrm{FeII}$ blend & 20041.1 & $2.3 \times 10^{-15}$ & -6.4 \\
$\mathrm{H}_{2}(1-0) \mathrm{S}(2)$ & 20338.0 & $2.4 \times 10^{-15}$ & -6.3 \\
$\mathrm{HeI}$ & 20587.0 & $1.3 \times 10^{-15}$ & -1.9 \\
$\mathrm{H}_{2}(2-1) \mathrm{S}(3)$ & 20730.0 & $3.1 \times 10^{-15}$ & -7.9 \\
$\mathrm{H}_{2}(1-0) \mathrm{S}(1)$ & 21218.3 & $6.8 \times 10^{-15}$ & -17.2 \\
$\mathrm{Br} 7(\mathrm{Br} \gamma)$ & 21661.3 & $5.4 \times 10^{-15}$ & -12.7 \\
$\mathrm{H}_{2}(1-0) \mathrm{S}(0)$ & 22235.0 & $1.9 \times 10^{-15}$ & -4.3 \\
$\mathrm{H}_{2}(1-0) \mathrm{Q}(1)$ & 24066.0 & $9.9 \times 10^{-15}$ & -16.4 \\
$\mathrm{H}_{2}(1-0) \mathrm{Q}(2)$ & 24134.0 & $2.5 \times 10^{-15}$ & -4.2 \\
$\mathrm{H}_{2}(1-0) \mathrm{Q}(3)$ & 24237.0 & $3.5 \times 10^{-15}$ & -5.7 \\
$\mathrm{H}_{2}(1-0) \mathrm{Q}(4)$ & 24375.0 & $2.6 \times 10^{-15}$ & -4.3 \\
\hline
\end{tabular}

being brighter in $\mathrm{H}_{2}$ molecular emission than $\mathrm{Br} \gamma$ emission. The spectrum shows a rising continuum towards longer wavelengths
Table 6. Identified lines of source I.

\begin{tabular}{lccc}
\hline \hline Line & $\begin{array}{c}\lambda \\
(\mu \mathrm{m})\end{array}$ & $\begin{array}{c}\text { Flux } \\
\left(\mathrm{erg} \mathrm{cm}^{-2} \mathrm{~s}^{-1}\right)\end{array}$ & $\begin{array}{c}\text { EW } \\
(\AA)\end{array}$ \\
\hline $\mathrm{KI}$ & 15163.1 & $1.3 \times 10^{-15}$ & -3.5 \\
$\mathrm{Br} 19$ & 15264.7 & $1.3 \times 10^{-15}$ & -3.6 \\
$\mathrm{FeI}$ & 15299.0 & $0.7 \times 10^{-15}$ & -2.1 \\
$\mathrm{Br} 18$ & 15346.0 & $0.7 \times 10^{-15}$ & -2.1 \\
$\mathrm{Br} 17$ & 15443.1 & $0.7 \times 10^{-15}$ & -1.9 \\
$\mathrm{Br} 16$ & 15560.7 & $1.5 \times 10^{-15}$ & -4.0 \\
$\mathrm{FeI}$ & 15697.0 & $1.2 \times 10^{-15}$ & -3.3 \\
$\mathrm{Br} 15$ & 15705.0 & $0.9 \times 10^{-15}$ & -2.5 \\
$\mathrm{MgI}$ & 15753.0 & $1.2 \times 10^{-15}$ & -3.6 \\
$\mathrm{MgI}+\mathrm{Br} 14$ & 15885.0 & $0.9 \times 10^{-15}$ & -2.7 \\
$\mathrm{SiI}+\mathrm{FeI}$ & 15964.0 & $1.5 \times 10^{-15}$ & -4.2 \\
$\mathrm{SiI}+\mathrm{Br} 13$ & 16099.0 & $4.4 \times 10^{-15}$ & -12.4 \\
$\mathrm{SiI}+\mathrm{FeI}$ & 16386.0 & $2.4 \times 10^{-15}$ & -7.1 \\
$\mathrm{FeII}$ & 16440.0 & $3.7 \times 10^{-15}$ & -10.8 \\
$\mathrm{Br} 11$ & 16811.2 & $4.6 \times 10^{-15}$ & -13.0 \\
$\mathrm{CI}$ & 16895.0 & $0.9 \times 10^{-15}$ & -2.6 \\
$\mathrm{MgI}$ & 17113.3 & $0.9 \times 10^{-15}$ & -2.6 \\
$\mathrm{Br} 10+\mathrm{NaI}$ & 17366.7 & $4.4 \times 10^{-15}$ & -11.7 \\
$\mathrm{H} 2(1-0) \mathrm{S}(7)$ & 17480.0 & $3.4 \times 10^{-15}$ & -8.9 \\
$\mathrm{H}$ (1-0) S(2) & 20338.0 & $2.7 \times 10^{-15}$ & -6.5 \\
$\mathrm{HeI}$ & 20587.0 & $10.1 \times 10^{-15}$ & -24.5 \\
$\mathrm{H} 2(2-1) \mathrm{S}(3)$ & 20730.0 & $5.0 \times 10^{-15}$ & -12.7 \\
$\mathrm{H} 2(1-0) \mathrm{S}(1)$ & 21218.3 & $12.0 \times 10^{-15}$ & -32.2 \\
$\mathrm{H} 2(2-1) \mathrm{S}(2)$ & 21542.0 & $1.4 \times 10^{-15}$ & -3.9 \\
$\mathrm{Br} 7(\mathrm{Br} \gamma)$ & 21661.3 & $24.0 \times 10^{-15}$ & -65.1 \\
$\mathrm{HeI}$ & 21840.0 & $1.8 \times 10^{-15}$ & -5.0 \\
$\mathrm{NaI}$ & 22062.4 & $2.4 \times 10^{-15}$ & -6.6 \\
$\mathrm{NaI}$ & 22089.7 & $3.4 \times 10^{-15}$ & -9.4 \\
$\mathrm{H}_{2}(1-0) \mathrm{S}(0)$ & 22235.0 & $3.0 \times 10^{-15}$ & -8.3 \\
$\mathrm{TiI}$ & 22280.1 & $3.6 \times 10^{-15}$ & -9.9 \\
$\mathrm{H}_{2}(2-1) \mathrm{S}(1)$ & 22477.0 & $2.2 \times 10^{-15}$ & -6.1 \\
$\mathrm{CaI}$ & 22614.1 & $5.2 \times 10^{-15}$ & -14.7 \\
$\mathrm{CaI}$ & 22657.3 & $46.0 \times 10^{-15}$ & -17.5 \\
$\mathrm{FeII}$ & 22727.3 & $4.0 \times 10^{-15}$ & -12.8 \\
$\mathrm{MgI}$ & 22814.1 & $2.3 \times 10^{-15}$ & -7.8 \\
\hline & & &
\end{tabular}

with $\mathrm{H}_{2}$ emission lines. The spectrum displays prominent $\mathrm{HeI}$ emission line at $2.058 \mu \mathrm{m}$, indicating that the source must have a temperature $>20000 \mathrm{~K}$ to excite this line. This emission is not extended (in contrast to $\mathrm{Br} \gamma$ or $\mathrm{H}_{2}$ ), therefore probably comes from the source itself, and suggests the source must be an early-type star. Trundle et al. (2007) approximate a spectral-type effective temperature relation at the SMC metallicity. In their schema, only stars with spectral types B3 or higher have temperatures high enough to excite the HeI $2.058 \mu \mathrm{m}$ line. The absence of any HeII lines along with the CIV 2.070$2.079 \mu \mathrm{m}$ lines suggests a later O-early or B spectral type. Nevertheless, the emission line spectra of such sources do not allow for a highly reliable classification using purely the MK scheme. Based on the approximate spectral type between late $\mathrm{O}(\mathrm{O} 7)$ and early B (B3) at SMC metallicity, we take the logarithm of the effective temperature $\left(\log T_{\text {eff }}\right)$ of the source to be $4.5_{-0.2}^{+0.1}$ (Trundle et al. 2007). The error on the temperature is due to the spectral typing error of six sub-types.

Source E. This source has the second brightest ISO peak (Contursi et al. 2000) and shows the most extreme NIR colours 
(Table 1). In general, the spectrum is featureless in comparison to Sources $\mathrm{C}$ or I, with molecular $\mathrm{H}_{2}$ and ionized hydrogen emission lines. The ratio of $\mathrm{H}_{2} 2.12 \mu \mathrm{m}$ to $\mathrm{Br} \gamma$ emission lines is higher than that of Source C. Also, the long-slit spectrum shows extended emission in $\mathrm{H}_{2}$ of about $3^{\prime \prime}$, and the Br $\gamma$ emission line is stronger on the source but fainter in the nebulosity. The $\log T_{\text {eff }}$ assuming a B0 spectral type at SMC metallicity is $4.45_{-0.2}^{+0.1}$ (Massey et al. 2005).

Source I. This source is located in an $\mathrm{H}_{2}$ knot (Rubio et al. 2000, Figs. 6 and 8), embedded in the head of a trunk-like feature pointing to the direction of the core of NGC 346. The NIR spectrum shows the $\mathrm{H}_{2}$, Hydrogen Brackett series, and HeI emission line at $\lambda 2.06 \mu \mathrm{m}$. The 2D image shows $\mathrm{Br} \gamma$ emission concentrated on the stellar source, while the $\mathrm{H}_{2}$ is extended and coming from the surrounding nebulosity. Like Source $\mathrm{C}$, temperatures $>20000 \mathrm{~K}$ are required to excite the helium gas. The presence of both HeI similar to Source $\mathrm{C}$ suggests a late $\mathrm{O}$ or early $\mathrm{B}$ spectral type. Following the prescription for Source C, we adopt $\log T_{\text {eff }}$ of $4.5_{-0.2}^{+0.1}$. The spectrum of ISO peak I shows strong PolyAromatic Hydrocarbon (PAH) typical of the mid-IR spectrum of a photo-dissociation region (PDR). We believe that the $\mathrm{H}_{2}$ emission is arising from the PDR (Whelan et al. 2013).

It should be noted that the LCO images show one source while the higher-resolution ISAAC/VLT images resolve the source into two components separated by $\sim 1^{\prime \prime}$. The spectrum shown in Fig. 3 corresponds to the brightest IR component. The other IR component in the slit is a field star with a spectrum (not shown) typical of a carbon star.

\subsection{Extinction}

We estimate the extinction of each source using the difference between the observed and intrinsic archival optical colours. We do not use the NIR colours as they are likely affected by strong IR excesses (see Sect. 3.1). The intrinsic colours are calculated by convolving the appropriate filter curves with Castelli \& Kurucz (2004) stellar models at $Z=0.004$. We set the luminosity classification to a dwarf star, that is, $\log g=4.0$. We note that the luminosity classification (except if the star is a supergiant) does not affect the stellar colour within the error of the spectral typing.

For Source C, we find $E(V-I)=1$. Based on the extinction coefficients of Schlegel et al. (1998), we calculate $A_{V}=2.5 \pm 1$, where the error arises from a combination of the error on the reddening due to the uncertainty in spectral type, and random photometric error. The nearest optical counterpart to Source E in Sabbi et al. (2007) is around 0'.2 from our NIR source, and does not seem to be physically related to it. Instead, we use the $J$-band magnitude to determine the extinction. We assume an intrinsic $J$ magnitude of a B0-B1 star (Pecaut \& Mamajek 2013), leading to $A_{J}=0.45 \pm 0.4$, where the error is purely due to the uncertainty in spectral type. This places the absolute extinction, $A_{V}=1.7 \pm 1$. Source I has $E(V-I)=0.5$, leading to an $A_{V}=$ $1.2 \pm 1$.

\subsection{Infrared SED}

The NIR and archival optical and IR photometry was used to construct the spectral energy distribution (SED) of each source (Fig. 4). The SED was then used to calculate the SED slope in the IR, using the formula,

$\alpha_{\mathrm{IR}}=\frac{d \log \left(\lambda F_{\lambda}\right)}{d \log \lambda}$, where $\lambda$ is between 3.6 and $24 \mu \mathrm{m}$, and is used to diagnose the evolutionary stage of the disk-star system Lada (1987). We use the classification scheme of Greene et al. (1994) to distinguish between systems with likely protostellar disks (Class I), optically thick circumstellar disks (Class II), or debris/transitional disk (Class III) objects. To calculate the SED slope, we fit the Spitzer [3.6], [4.5], [5.0], [8.0], and $24 \mu \mathrm{m}$ fluxes calculated from their unreddened magnitudes. We used the excess ratios from Sung et al. (2009) to derive the extinction coefficients in the Spitzer bands. The resultant $\alpha_{\mathrm{IR}}$ values of all stars fall above 0.1 , classifying them as Class I sources. The SEDs beyond $\lambda>24 \mu \mathrm{m}$ are dominated by dust, which must have been evacuated in the immediate vicinity of the star or be in the form of a circumstellar disc for the central star to be optically visible.

In addition, we fit the IR SED to the models of Robitaille et al. (2006). The model grid covers 20000 radiative transfer YSO models across a mass range of $0.1-50 M_{\odot}$. From these fits, we can estimate the approximate stage of a YSO, as well as its mass and approximate luminosity with the associated fitting errors. We find that the best fit result $\left(\chi^{2} /\right.$ datapoint $\left.<4\right)$ for Source C corresponds to a Stage I YSO, with a mass of $18 M_{\odot}$. Simon et al. (2007) classified the same source based purely on the mid-IR photometry from Spitzer and found a fit consistent to either an AGB star, or a YSO, favouring the latter. For Source E, the best fit results in Stage I YSO, with a mass of $17 M_{\odot}$ closely matching that from Simon et al. (2007). The bestfit for Source I is extremely poor $\left(\chi^{2} /\right.$ datapoint $\left.\sim 100\right)$, and we do not consider it a valid representative of the SED. It is shown for completeness. We stress that the SED fits are not intended to provide accurate parameters, but to be a crude guide to the nature of each source. Furthermore, these models are limited in nature compared to the available free parameters (Offner et al. 2012).

\section{5. $\mathrm{H}_{2}$ line ratios}

Molecular $\mathrm{H}_{2}$ NIR emission in YSOs is caused either by (i) collisional excitation caused by outflows or shock from the YSOs, or (ii) UV radiation from nearby OB stars forming a photodissociation region (PDR). The flux-calibrated line ratios are an important diagnostic tool to differentiate between collisional excitation and fluorescence in PDR regions (Black \& van Dishoeck 1987; Sternberg \& Dalgarno 1989; Draine \& Bertoldi 1996; Burton et al. 1990, 1998). In the case of collisional excitation, the temperature of the gas is typically of the order of 2000-3000 K, resulting in only the lower levels of $\mathrm{H}_{2}$ being populated. In the case of UV fluorescence, however, the $\mathrm{H}_{2}$ molecule is lifted into high and low states by absorption of high-energy UV photons which subsequently decay. Therefore, in the cases of collisional excitation, there is an absence of transitions from high levels and a high ratio $(>10)$ of fluxes in the $2.12 \mu \mathrm{m}$ 1-0 $\mathrm{S}(1)$ to the $2.24 \mu \mathrm{m} \mathrm{2-1} \mathrm{S}(1)$ lines, and a low ratio $(<1)$ in the $2.22 \mu \mathrm{m} \mathrm{1-0} \mathrm{S(0)} \mathrm{to} \mathrm{the} 2.12 \mu \mathrm{m} \mathrm{1-0} \mathrm{S(1).} \mathrm{On} \mathrm{the}$ contrary, in the case of UV fluorescence, there is a low ratio of fluxes $(<2)$ in $2.12 \mu \mathrm{m} \mathrm{1-0} \mathrm{S(1)} \mathrm{to} \mathrm{the} 2.24 \mu \mathrm{m} \mathrm{2-1} \mathrm{S(1),} \mathrm{and} \mathrm{a}$ high ratio $(>1)$ in $2.22 \mu \mathrm{m} \mathrm{1-0} \mathrm{S(0)} \mathrm{to} \mathrm{the} \mathrm{2.12} \mu \mathrm{m} \mathrm{1-0} \mathrm{S(1).}$

The $\mathrm{H}_{2}$ line fluxes for the three transitions given in Tables 4-6 show that these are consistent with models for dense PDRs. In source $C$, where we have the best $\mathrm{S} / \mathrm{N}$, we are dominated by an approximate pure fluorescence, dense PDR regime, although we cannot discard the possibility of collisional excitation. In Source $\mathrm{E}$, the non-detection of the $\mathrm{H}_{2}$ emission at $2.24 \mu \mathrm{m}$ puts a lower limit on the ratio of this transition. Given this fact, the $\mathrm{H}_{2}$ emission would also originate in dense PDRs with a greater degree of local extinction (ratio increases due to 

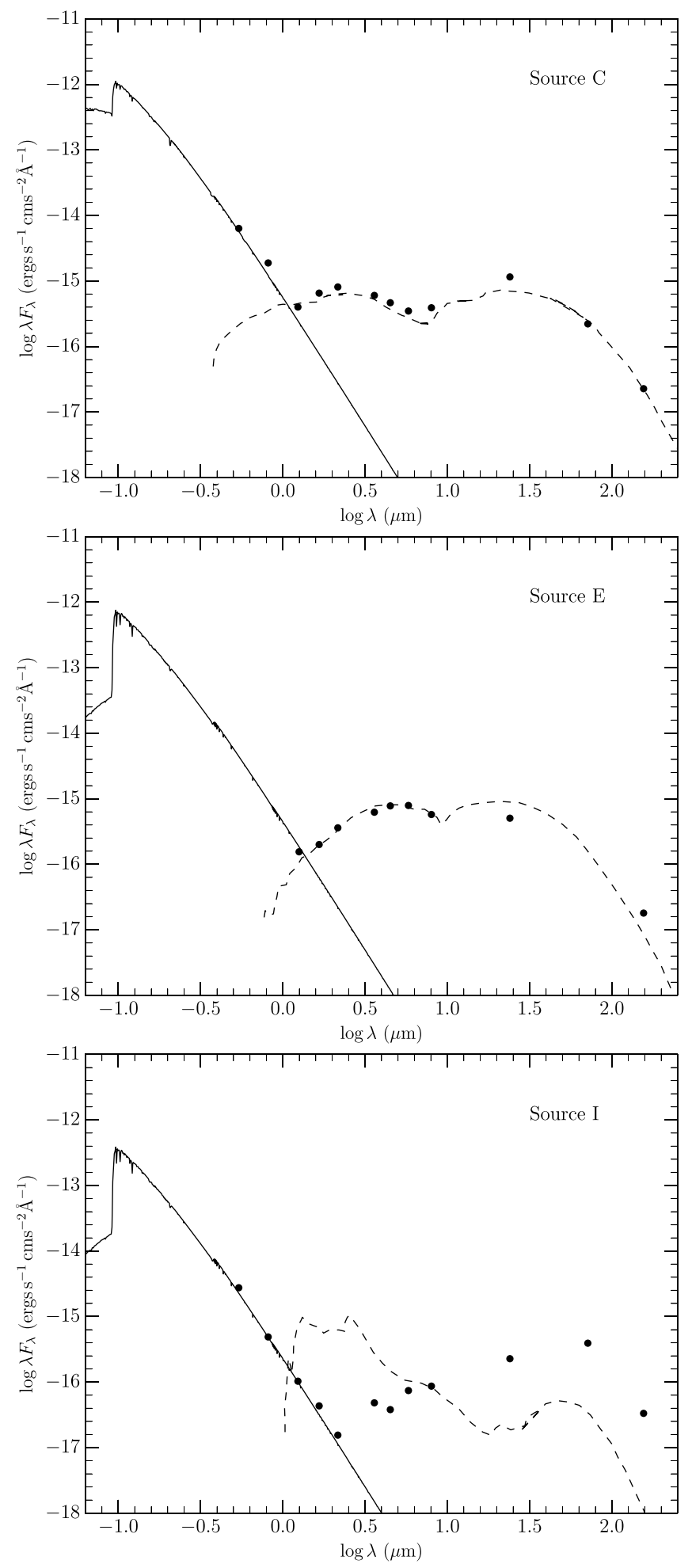

Fig. 4. Optical $(0.55 \mu \mathrm{m})$-mid-IR $(160 \mu \mathrm{m})$ SED of each of the sources. The overplotted stellar spectra (solid lines) are $1 / 5 Z_{\odot}$ Castelli \& Kurucz (2004) models at the nearest $T_{\text {eff }}$ to that derived in Sect. 3.1. The best fitting YSO model from Robitaille et al. (2006) is also overplotted as dashed lines. The dots mark the photometry from Tables $2 \& 3$, corrected for extinction values estimated in Sect. 5.

the increased intensity of $\mathrm{H}_{2} 2.22 \mu \mathrm{m}$ ) although we cannot rule out the presence of excitation by unresolved shocks in the same area.

Excitation by shock is expected to happen very close to the mYSOs, at scales of $0.1-0.2 \mathrm{pc}$. While further away, the dominant mechanism should be the UV radiation from the surrounding OB association (Israel \& Koornneef 1988). Thus, it is very difficult to spatially resolve the $\mathrm{H}_{2}$ emission caused by shock or fluorescence at the distance of the SMC, where $1^{\prime \prime}=0.3 \mathrm{pc}$. We are most probably in the presence of a combined contribution of emission mechanisms and therefore can only consider the dominant one. In the case of source $\mathrm{C}$, where the spectrum shows the best $\mathrm{S} / \mathrm{N}$, the $\mathrm{H}_{2}$ emission extends $2^{\prime \prime} .5$ to each side of the source $(0.8 \mathrm{pc})$ which would indicate the existence of a cocoon with fluorescence emission. The source $\mathrm{C}$ spectrum has $\mathrm{HeI}$ lines meaning that it can provide the necessary UV photons needed to form an internal PDR in the cocoon. Nevertheless, we have to consider that source $C$ is located near the core of the NGC 346 cluster, which contains tens of massive stars, and thus could also contribute to the PDR $\mathrm{H}_{2}$ emission.

\section{Discussion}

\subsection{Evolutionary status}

The evolutionary status of our stars is discerned using their positions in the Hertzsprung-Russell diagram (HRD), their IR excess and SED, and their spectral signatures.

Firstly, we place them in the Hertzsprung-Russell diagram in Fig. 5. To calculate the luminosity, we applied bolometric corrections for the $J$ band calculated from the appropriate Castelli \& Kurucz (2004) model on the extinction and distance-corrected $J$-band magnitude. The uncertainty on the luminosities includes the uncertainty in the extinction and spectral classification. We overplot PMS single-star isochrones and stellar mass tracks at $Z=0.004$ (Bressan et al. 2012) up to the main-sequence phase on the HRD. Also shown are the locations of known mYSOs in the Galaxy, and the SMC.

The three sources lie above the main sequence, and between the 10000 and 50000 year isochrones, with estimated logarithmic ages $\log t$ of $4.25 \pm 1.0,4.51 \pm 0.6$, and $4.44 \pm 1.0 \mathrm{yr}$ for sources $\mathrm{C}, \mathrm{E}$, and $\mathrm{I}$, respectively, from interpolation between the isochrones. These sources fall in the plane occupied by the brightest known SMC YSOs (Sewiło et al. 2013). Compared to the Galactic YSOs, for example in W49, they are more luminous than their exact spectral type counterparts, although none are as massive as the $>50 M_{\odot}$ YSOs reported in that region (Wu et al. 2016). Interpolating from the mass tracks, we estimate that source $\mathrm{C}$ is the most massive, at around $26.0 \pm 5.0 M_{\odot}$, and $\mathrm{E}$ and $\mathrm{I}$ at $15.9 \pm 3.0 M_{\odot}$ and $19.7 \pm 4.0 M_{\odot}$ respectively. We note the precise values of the masses and ages derived using both the isochrones and SED model fits are uncertain, and only provide an approximate estimate of both parameters. The final stellar parameters are displayed in Table 7.

The $3 \mu \mathrm{m}-24 \mu \mathrm{m}$ SED slope of all three sources fall within Class I YSO classification, suggesting an IR disc peaking at $\lambda>100 \mu \mathrm{m}$, indicative of strong IR excess. In addition, we fit the photometry with wavelengths greater than $3 \mu \mathrm{m}$ with the YSO models from Robitaille et al. (2006; Fig. 5). Sources C and $\mathrm{E}$ have good fits (with $\chi^{2} /$ datapoint $<4$ ) for young massive YSOs, although Source I has a poor fit. These IR characteristics are unique to YSOs, with the exception of $\mathrm{B}[\mathrm{e}]$ stars that are a heterogeneous group of stars with IR excesses similar to Class II YSOs, but $\mathrm{B}[\mathrm{e}]$ stars have metal line emission (Liermann et al. 2014) in their spectra which are not seen in these spectra. All three spectra show Bry emission close to the source, possibly from mass being accreted from a circumstellar disc, although the combination of the poor resolution and low S/N do not allow for a detailed examination of the line 


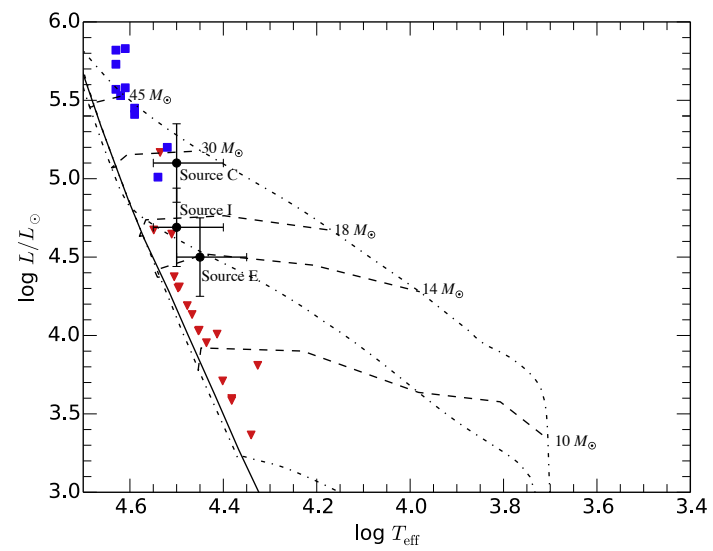

Fig. 5. Positions of our mYSO candidates (circles) are shown along with known SMC YSOs (Sewiło et al. 2013) as red inverted triangles and galactic YSOs (Wu et al. 2016) as blue squares. The dashed dotted lines are the PMS isochrones of (Bressan et al. 2012) at $1 / 5 Z_{\odot}$ for 0.01 , 0.03 , and $0.3 \mathrm{Myr}$ right to left, respectively, and the dashed lines are the stellar tracks for $10,14,18,30$, and $45 M_{\odot}$ from bottom up. The solid line is the main sequence locus.

Table 7. Stellar parameters of the mYSOs.

\begin{tabular}{lcccc}
\hline \hline Star & $\begin{array}{c}T_{\text {eff }} \\
(\mathrm{K})\end{array}$ & $\begin{array}{c}\log L_{*} \\
\left(L_{\odot}\right)\end{array}$ & $\begin{array}{c}\text { Mass } \\
\left(M_{\odot}\right)\end{array}$ & $\begin{array}{c}\log \text { Age } \\
(\mathrm{yr})\end{array}$ \\
\hline Source C & $4.5_{-0.2}^{+0.1}$ & $5.1 \pm 0.25$ & $26 \pm 5$ & $4.25 \pm 1.0$ \\
Source E & $4.45_{-0.2}^{+0.1}$ & $4.5 \pm 0.25$ & $15.9 \pm 3.0$ & $4.51 \pm 0.6$ \\
Source I & $4.5_{-0.2}^{+0.1}$ & $4.69 \pm 0.25$ & $19.7 \pm 4.0$ & $4.44 \pm 1.0$ \\
\hline
\end{tabular}

profiles. In addition, all the sources show extended $\mathrm{H}_{2}$ emission, arising mostly due to fluorescence, but there could also be a possible contribution from shocked gas from outflows. All these characteristics point unambiguously towards a classification as mYSOs.

\subsection{Comparison with literature classifications}

Two major YSO IR studies have been conducted using data from the Spitzer telescope which include our three sources: Sewiło et al. (2013) and Simon et al. (2007).

Sewiło et al. (2013) classified Spitzer YSO candidates in the SMC using 3.6-24 $\mu \mathrm{m}$ photometry. Their classification was based on the position of each candidate in various IR CMD and CCD, which allowed them to differentiate them from contaminants. Based on their positions in multiple diagrams, each candidate was given a score indicating the likelihood of being a YSO. In addition, they also fit SED models to their photometry, however none of the SED fits in Sewiło et al. (2013) meeting their $\chi^{2}$ requirement include our sources. Instead, sources C (Y535) and E (Y540) are both classified as possible YSO candidates based on their CMD scores, while source I (Y552) is classified as YSO, and is flagged as likely having polyaromatic hydrocarbon (PAH) emission based on its SED fit, which contributes to its poor fit score. The emission is confirmed in the study done by Whelan et al. (2013) using Spitzer mid-IR spectra.

Simon et al. (2007) catalogued a list of YSOs in N66 based on the Spitzer photometry and modelling adjusted to the obtained SEDs. Source E is the brightest source of their catalogue (their source 25), with a luminosity of $30300 L_{\odot}$ and a stellar mass of $16.6 M_{\odot}$. The source is classified as Stage I type, following the classification scheme defined by Robitaille et al. (2006). Source C is mentioned as the fourth most luminous object at $8 \mu \mathrm{m}$, that is, SSTS3MC 14.7725-72.1766, but they do not classify it as a YSO as they could fit its SED either with a YSO or an AGB SED. They favour the possibility of it being a YSO with a luminosity of $3.3 \times 10^{4} L_{\odot}$ and mass of $14.7 M_{\odot}$, mainly based on the fact that the source is located in the center of NGC 346 and surrounded by many young massive stars. The luminosities of both sources from Simon et al. (2007) fall within the error ranges of the luminosities derived in our work, however only the mass of source $\mathrm{E}$ matches our derived mass within error estimates, whereas for Source C, we find a mass $\sim 10 M_{\odot}$ higher than that estimated from SED fitting in Simon et al. (2007). From the spectrum alone based on the HeI line, we suggest that $C$ should be much more massive than derived in Simon et al. (2007). Source I is not in their catalogue.

\subsection{Formation scenario}

The molecular gas observations by Rubio et al. (2000) and the mid-IR ISO observations by Contursi et al. (2000) suggested that the dense clumps of neutral hydrogen gas and dust in the N 66 HII region could be the sites of recent star formation. The IR spectra obtained towards Sources C, E, and I in N 66 plus their NIR colours confirm that these sources are embedded mYSOs with characteristics of the Class I type, thereby confirming that these dense clumps harbour YSOs, and are the sites of recent star formation. They are located in regions of high density of neutral molecular gas as seen in the $\mathrm{CO}(2-1)$ emission line and are associated to $\mathrm{H}_{2}$ knots as seen in the $\mathrm{H}_{2}$ images as well as spatially resolved emission in the long-slit NIR spectra.

From the derived properties of the sources, we find that source $\mathrm{C}$ is the most massive with a mass $>25 M_{\odot}$, whereas sources $\mathrm{E}$ and $\mathrm{I}$ are almost at similar ages, with masses around $\sim 15-20 M_{\odot}$. This suggests a different scenario to the sequential star-formation scenario of Contursi et al. (2000) and Rubio et al. (2000), where the authors suggest that star formation has begun at NGC 346 and continues outward along the bar. Instead, the youngest and most massive YSO is found in the central cluster, while comparatively older and less massive YSOs are found along the bar.

Although, the low numbers do not enable any statistical study for age spreads or a formation scenario, we would argue that star formation at least in the central cluster NGC 346 seems to have been ongoing. Source $\mathrm{C}$, which has moderate extinction of $A_{V} \sim 2.5$, is not located behind the molecular clump (which would suggest an extinction $>8$ ), so it should lie at the cluster, or between cluster and our line of sight. This source is therefore most likely part of the cluster, which has a published age of 3 Myr (Sabbi et al. 2007) based on isochrone fitting. De Marchi et al. (2013) find a much younger PMS population identified based on their $\mathrm{H} \alpha$ excess emission of around $1 \mathrm{Myr}$, which is more in line with the age we estimate for Source C. The presence of a mYSO at the very heart of the cluster suggests that it must have formed there, indicating that star formation has either been on-going continuously, or has undergone another very recent burst since the formation of the cluster four million years ago. It is interesting to note that the $\mathrm{H} \alpha$ HST image shows a source associated to the NIR source C. In fact, all our information suggests that source $\mathrm{C}$ is a complex source, and is likely a mYSO enshrouded in a compact HII region excited by $\mathrm{O}$ stars that ionize the hydrogen gas around them. The detected $\mathrm{H}_{2}$ lines could be produced on the interface of the PDR where the neutral gas is being excited by these young 
stars. Molecular gas is present in the region although we do not presently have a high-resolution CO map towards NGC 346 to show the molecular cloud structure. This structure most probably would consist of dense and small CO cores such as those seen with ALMA arcsecond-resolution CO images near 30 Doradus (Indebetouw et al. 2013) or in WLM (Rubio et al. 2015). Further, arcsecond-resolution molecular maps are necessary to confirm this hypothesis.

\section{Conclusions}

We present $H K$ spectra of three bright sources selected using NIR $J H K$ photometry in the N 66/NGC 346 region of the SMC. Based on a combination of the spectral features seen in the NIR, photometry, and SED we suggest that sources C and I are of early spectral type, between late $O$ and early B. Source $E$ is more likely an early B star. This spectral classification provides a crude estimate of the effective temperature and extinction of the sources which allows us to place them in the HRD. Based on their positions in the HRD, we would suggest that they have ages between 10000 and 50000 yr. The evolutionary mass of Source $\mathrm{C}$ would be $\sim 25 M_{\odot}$, while that of Sources $\mathrm{E}$ and I is between 15 and $20 M_{\odot}$. The SEDs of each source show excess longwards of $\lambda>1 \mu \mathrm{m}$. Both their SED slopes and results from fitting against the YSO models of Robitaille et al. (2006) suggest that all three sources are likely mYSOs. The nature of source $\mathrm{C}$ is more complex compared to sources $\mathrm{E}$ and I given its high NIR luminosity and position near the centre of NGC 346, and further study of this source is necessary. Combining all the information at our disposal, we suggest that sources observed here are mYSOs in N 66. The NIR spectroscopy combined with NIR to mid-IR photometry, confirms that a new generation of stars is being formed in the dense clumps in the neutral molecular gas of N 66. The interaction of the massive stars in NGC 346 with the surrounding molecular gas may have created the conditions for a new star formation event.

Acnowledgements. We would like to thank the anonymous referee for his/her valuable comments and careful reading of the manuscript. M.R. wishes to acknowledge support from CONICYT (CHILE) through FONDECYT grant No 1140839 and partial support through project BASAL PFB-06. R.B. wishe to acknowledge support from FONDECYT (CHILE) grant No 1050052, and DIULS CD 08102, and V.M.K acknowledges support from the FONDECYT Postdoc Fellowship grant No 3160117.

\section{References}

Bik, A., Kaper, L., \& Waters, L. B. F. M. 2006, A\&A, 455, 561

Black, J. H., \& van Dishoeck, E. F. 1987, ApJ, 322, 412

Blum, R., Daminelli, A., Conti, P., et al. 2001, AJ, 121, 3149
Bressan, A., Marigo, P., Girardi, L., et al. 2012, MNRAS, 427, 127

Burton, M. G., Geballe, T. R., Brand, P. W. J. L., \& Moorhouse, A. 1990, ApJ, 352,625

Burton, M. G., Howe, J. E., Geballe, T. R., \& Brand, P. W. J. L. 1998, PASA, 15, 194

Castelli, F., \& Kurucz, R. L. 2004, ArXiv e-prints [arXiv:astro-ph/0405087]

Contursi, A., Lequeux, J., Cesarsky, D., et al. 2000, A\&A, 362, 310

De Marchi, G., Beccari, G., \& Panagia, N. 2013, ApJ, 775, 68

Draine, B. T., \& Bertoldi, F. 1996, ApJ, 468, 269

Eiroa, C., Oudmaijer, R. D., Davies, J. K., et al. 2002, A\&A, 384, 1038

Felli, M., Massi, M., Staude, H. J., et al. 1984, A\&A, 135, 261

Gouliermis, D. A., Dolphin, A. E., Brandner, W., \& Henning, T. 2006, ApJS, 166,549

Gouliermis, D. A., Bestenlehner, J. M., Brandner, W., \& Henning, T. 2010, A\&A, 515, A56

Greene, T. P., Wilking, B. A., Andre, P., Young, E. T., \& Lada, C. J. 1994, ApJ, 434, 614

Hanson, M. M., Howarth, I. D., \& Conti, P. S. 1997, ApJ, 489, 698

Henize, K. G. 1956, ApJS, 2, 315

Hilditch, R. W., Howarth, I. D., \& Harries, T. J. 2005, MNRAS, 357, 304

Indebetouw, R., Brogan, C., Chen, C.-H. R., et al. 2013, ApJ, 774, 73

Israel, F. P., \& Koornneef, J. 1988, A\&A, 190, 21

Kalari, V. M., \& Vink, J. S. 2015, ApJ, 800, 113

Kalari, V. M., Vink, J. S., Dufton, P. L., et al. 2014, A\&A, 564, L7

Kalari, V. M., Rubio, M., Elmegreen, B. G., et al. 2018, ApJ, 852, 71

Keller, S. C., \& Wood, P. R. 2006, ApJ, 642, 834

Lada, C. J. 1987, Star Forming Regions, 115, 1

Lenorzer, A., Bik, A., de Koter, A., et al. 2004, A\&A, 414, 245

Liermann, A., Schnurr, O., Kraus, M., et al. 2014, MNRAS, 443, 947

Massey, P., Parker, J. W., \& Garmany, C. D. 1989, AJ, 98, 1304

Massey, P., Puls, J., Pauldrach, A. W. A., et al. 2005, ApJ, 627, 477

Nota, A., Sirianni, M., Sabbi, E., et al. 2006, ApJ, 640, L29

Offner, S. S. R., Robitaille, T. P., Hansen, C. E., McKee, C. F., \& Klein, R. I 2012, ApJ, 753, 98

Oliveira, J. M., van Loon, J. Th., Stanimirović, S., \& Zijlstra, A. A. 2006, MNRAS, 372, 1509

Pecaut, M. J., \& Mamajek, E. E. 2013, ApJS, 208, 9

Persson, S. E., West, S. C., Carr, D. M., et al. 1992, PASP, 104, 204

Robitaille, P. P., Whitney, B. A., Indebetow, R., Wood, K., \& Denzmore, P. 2006, ApJS, 167, 256

Rubio, M., Barbá, R. H., Walborn, N., et al. 1998, AJ, 116, 1708

Rubio, M., Contursi, A., Lequeux, J., et al. 2000, A\&A, 359, 1139

Rubio, M., Elmegreen, B. G., Hunter, D. A., et al. 2015, Nature, 525, 218

Russell, S. C., \& Dopita, M. A. 1992, ApJ, 384, 508

Sabbi, E., Siriani, M., Nota, A., et al. 2007, AJ, 133, 44

Seale, J. P., Meixner, M., Sewiło, M., et al. 2014, AJ, 148, 124

Sewiło, M., Carlson, L. R., Seale, J. P., et al. 2013, ApJ, 778, 15

Simon, J., Bolatto, A., Whitney, B., et al. 2007, ApJ, 669, 327

Schlegel, D. J., Finkbeiner, D. P., \& Davis, M. 1998, ApJ, 500, 525

Schmeja, S., Gouliermis, D. A., \& Klessen, R. S. 2009, ApJ, 694, 367

Sternberg, A., \& Dalgarno, A. 1989, ApJ, 338, 197

Stetson, P. B. 1987, PASP, 99, 191

Sung, H., Stauffer, J. R., \& Bessell, M. S. 2009, AJ, 138, 1116

Trundle, C., Dufton, P. L., Hunter, I., et al. 2007, A\&A, 471, 625

van Loon, J. Th., Oliveira, J. M., Wood, P. R., et al. 2005, MNRAS, 364, L71

van Loon, J. Th., Cohen, M., Oliveira, J. M., et al. 2008, A\&A, 487, 1055

Ward, J. L., Oliveira, J. M., van Loon, J. T., \& Sewiło, M. 2017, MNRAS, 464, 1512

Whelan, D. G., Lebouteiller, V., Galliano, F., et al. 2013, ApJ, 771, 16

Wu, S.-W., Bik, A., Bestenlehner, J. M., et al. 2016, A\&A, 589, A16 